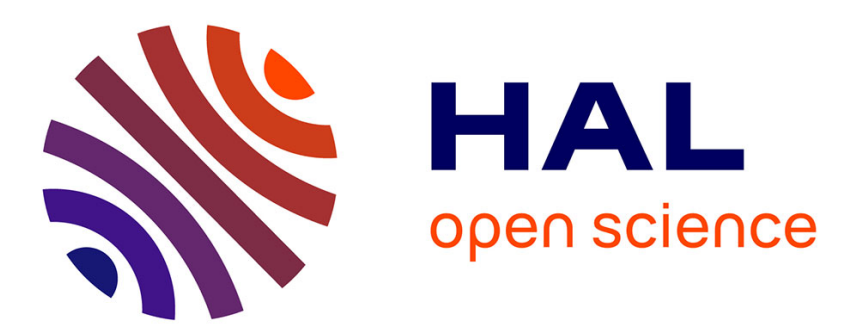

\title{
Les finances solidaires et l'économie sociale en roumanie: une réponse de "proximités" à la régionalisation d'une économie en transition?
}

Pascal Glemain, Emmanuel Bioteau, Alexandru Dragan

\section{- To cite this version:}

Pascal Glemain, Emmanuel Bioteau, Alexandru Dragan. Les finances solidaires et l'économie sociale en roumanie: une réponse de "proximités" à la régionalisation d'une économie en transition?. Annals of Public and Cooperative Economics, 2013, 84 (2), pp.195 - 217. 10.1111/apce.12009 . hal-01892571

\section{HAL Id: hal-01892571 \\ https://hal.univ-rennes2.fr/hal-01892571}

Submitted on 10 Oct 2018

HAL is a multi-disciplinary open access archive for the deposit and dissemination of scientific research documents, whether they are published or not. The documents may come from teaching and research institutions in France or abroad, or from public or private research centers.
L'archive ouverte pluridisciplinaire HAL, est destinée au dépôt et à la diffusion de documents scientifiques de niveau recherche, publiés ou non, émanant des établissements d'enseignement et de recherche français ou étrangers, des laboratoires publics ou privés. 


\title{
LES FINANCES SOLIDAIRES ET L'ÉCONOMIE SOCIALE EN ROUMANIE: UNE RÉPONSE DE «PROXIMITÉS» À LA RÉGIONALISATION D’UNE ÉCONOMIE EN TRANSITION?
}

\author{
par \\ Pascal GLEMAIN* \\ ESSCA CeRESS et CIAPHS Université de Rennes 2, France
}

\author{
Emmanuel BIOTEAU \\ Université d'Angers, France
}

et

\begin{abstract}
Alexandru DRAGAN
Université d'Angers, France et Université de l'Ouest de Timisoara, Roumanie
\end{abstract}

\begin{abstract}
RÉSUMÉ: La Roumanie apparaît en Europe centrale et orientale comme un territoire d'expérimentation d'une forme particulière d'économie sociale liant coopératives et organisations «not-for-profit». En transition économique et sociale, la Roumanie est également un pays en transition territoriale dont les systèmes de financements locaux ont quitté leur approche centralement planifiée, pour rejoindre un secteur financier solidaire dont les Instituts de Microfinance (IMFs) sont les principaux acteurs. La question à laquelle cet article tente d'apporter quelques éléments de réponse est celle du rôle de la finance solidaire locale dans la soutenabilité des transitions socio-économiques territoriales en cours. Il s'agit d'envisager une économie humaine au sein de laquelle les conditions dignes de vie, nous amène à envisager un modèle particulier d'économie conviviale située à partir des outils conviviaux des finances solidaires locales.
\end{abstract}

Keywords: Romania, Social Finance, Regionalisation Process, Social Economy.

\section{Social finance and social economy in Romania. A 'proximities' Issue for the regionalisation process of a west european country in transition?}

Romania seems to be a singular experimented space in the Central and Oriental Countries for Social Economy in the EU. Indeed, Romania tries to develop a social-base economic model through its

\footnotetext{
E-mails: pascal.glemain@wanadoo.fr; emmanuel.bioteau@univ-angers.fr; alexandru. dragan@etud.univ-angers.fr 
cooperative-based firms and its Not-For-Profit Organizations. In its social and economic transition process, Romania appears like a country where its local-based banking system is concerned more and more with the microfinance institutes. This paper wants to show the capabilities from socialbased finance to work about a local social-based sustainable development. That means that we are in front of a new human side model of development, where human dignity and capabilities are the main issues for a new convivialism model.

\section{Soziale Finanzwirtschaft und Sozialwirtschaft in Rumänien. Eine „Nähe-Beziehung” als Ansatz im Regionalisierungsprozess eines West Europäischen Landes in Transformation}

Rumänien scheint ein einzigartiges Experimentierfeld für Sozialwirtschaft in den zentralen und östlichen Ländern der EU zu sein. Es ist tatsächlich so, dass Rumänien versucht, mit seinen genossenschaftlichen Unternehmen und seinen Non Profit-Organisationen ein sozial- basiertes Wirtschaftsmodell zu entwickeln. In seinem sozialen und ökonomischen Transformationsprozess erscheint Rumänien als ein Land, in dem das örtlich verankerte Bankensystem sich mehr und mehr aus Mikrofinanzinstituten zusammensetzt. Mit dieser Untersuchung sollen die Fähigkeiten sozial-basierter Finanzwirtschaft aufgezeigt werden, eine örtliche sozial-basierte nachhaltige Entwicklung einzuleiten. Das bedeutet, sich an der Spitze eines neuen human ausgerichteten Entwicklungsmodells zu befinden, bei dem Menschenwürde und menschliche Fähigkeiten die Schwerpunkte eines neuen konvivialen Wirtschaftsmodells sind.

\section{Las finanzas solidarias y la economía social en Rumanía ¿Una respuesta de proximidad a la regionalización de una economía en transición?}

Rumanía se presenta en Europa central y oriental como un territorio de experimentación, como una forma particular de economía social ligada a las cooperativas y a las organizaciones sin ánimo de lucro. En transición económica y social, Rumanía es igualmente un país en transición territorial, cuyos sistemas de financiación local han abandonado su enfoque de planificación central, para asimilarse a un sector financiero solidario en el que los actores principales son los Institutos de Microfinanzas (IMF). La cuestión a la que trata de dar respuesta este artículo es la relativa al papel de la financiación solidaria local en la sostenibilidad de las transiciones socioeconómicas territoriales en curso. Se trata de considerar una economía humana en el seno de la cual las condiciones de vida digna nos lleven a proyectar un modelo particular de economía, concebido a partir de herramientas tales como las finanzas solidarias locales.

\section{La Roumanie: une économie d' Europe Centrale encore en transition?}

Malgré une croissance de l'ordre de $2 \%$ en 2011, la Roumanie sort d'une période de très forte récession ( $-1,5 \%$ en 2010 , et $-7 \%$ en 2011$)$. Dans ce contexte, à l'instar des autres pays d'Europe Centrale et Orientale en proie à de profondes mutations structurelles depuis les années 1990, leur transition d'un modèle d'économie centralement planifiée vers celui d'une économie de marché interroge. En effet, ce processus semble avoir pour conséquence un accroissement des inégalités socio-économiques et territoriales, d'où résultent des poches de pauvreté de plus en plus prégnantes en Europe. 
Selon Eurostat, en 2010, l'Union européenne comptait près de 18 millions de personnes pauvres (seuil à $60 \%$ du revenu national médian), ${ }^{1}$ soit plus de $16 \%$ de sa population totale. Parmi les taux les plus élevés - établis à plus de $20 \%$ - nous trouvons la Bulgarie et la Roumanie. Même s'il convient d'interpréter avec prudence ces chiffres car, comme le souligne Eurostat (2010): «Le seuil retenu n'est pas le revenu médian européen, mais le revenu médian de chaque pays»; il n'en demeure par moins, toujours selon Eurostat que: "Le Royaume-Uni enregistre 17,1\% de pauvres, mais le seuil de pauvreté britannique est beaucoup plus élevé que le seuil roumain si l'on considère cette donnée en valeur absolue : il est estimé à 853 euros par mois pour le Royaume-Uni contre 176 euros pour la Roumanie». Pour autant, pour citer Nussbaum (2012), bien qu'en nous intéressant à la Roumanie: «si le PIB d'un pays augmente chaque année et que le pourcentage de personnes privées d'instruction et de soins médicaux grandit lui aussi, ce pays est-il vraiment en progrès?»

En outre, la Roumanie subit depuis 2002 une émigration massive de sa jeune population, avec un solde négatif de 2,6 millions de personnes, et encore: le décalage étant beaucoup plus large si l'on parle de résidents effectifs en Roumanie. La baisse de population relève d'enjeux complexes, liés à la fois aux problèmes économiques et sociaux de la transition (à partir de 1990), mais aussi à l'émigration qui comme toujours affecte plus particulièrement les classes d'âges les plus jeunes, et accroît les problèmes de vieillissement. Le nombre de familles séparées par le départ de l'un voire des deux membres du couple ne cesse de s'accroître. Il n'est pas rare jusqu'en 2010 de rencontrer des grands-parents ayant la charge de leurs petits-enfants, leurs parents étant partis à l'étranger. Ainsi, les jeunes étant partis, malgré une légère reprise à la hausse de la natalité entre 2007 et 2009, en 2010 pour la première fois depuis la fin de la Seconde guerre mondiale moins de 200.000 nouveaux nés sont enregistrés. Surtout, depuis 2010 toujours, l'on constate une vague de rapatriement des enfants en bas âge par leurs parents... dans leur pays d'accueil de travail.

En d'autres termes, la vie de leurs ressortissants est-elle aussi digne ${ }^{2}$ qu'ils le souhaiteraient? Les moyens de financement dont ils disposent leur permettent-ils de répondre à leur «liberté substantielle» au sens de Sen (1990), cette «possibilité créée par la combinaison des capacités personnelles et d'un environnement politique, social et économique» (Nussbaum 2012, p. 39)?

En ce qui concerne le contexte macroéconomique roumain, notons que malgré l'augmentation continue de la dette publique qui atteint $35 \%$ du PIB en 2011, son déficit budgétaire reste contenu à $20 \%$ de celui-ci. La dette publique brute totale se situe à hauteur de 98,605 Mds € en décembre 2011 (BNR, 2012), soit un nouveau record historique et ce, bien que la Roumanie dispose encore par cette dette de la plus faible dette extérieure par habitant de l'UE, soit environ $4.500 €$ (Eurostat, 2012). Sur le fond, ce

1 Les taux de pauvreté correspondent à une proportion de la population totale qui vit avec un revenu disponible inférieur au seuil de pauvreté. Celui-ci correspond à un pourcentage du revenu médian national, celui qui sépare la population en deux, la moitié recevant moins que ce revenu, l'autre plus.

2 Nussbaum (2012, p. 51) définit la dignité comme «relative à la notion de respect (...). L'idée fondamentale est que certaines conditions de vie donnent aux individus une vie qui est digne de l'être humain qu'ils sont et d'autres non. Dans ce dernier cas, ils conservent leur dignité mais elle est un chèque sans provision». 
budget s'avère être insuffisant pour la mise en paiement des salaires de la fonction publique et des pensions. En conséquence la Roumanie a vu sa dette publique doubler entre 2008 et 2010 en raison de la nécessité d'un soutien financier du FMI, de la Commission européenne et de banques privées. Ces dernières possèdent actuellement $66,7 \%$ du total des créances, qu'elles fixent à des taux d'intérêt plus élevés que ceux pratiqués par les établissements internationaux d'appui aux Etats. Il en résulte au niveau national un «creux bancaire», c'est-à-dire un retrait des banques locales du financement local qui fragilise son modèle de développement local. Le sous-investissement local induit accroit les conséquences du tassement significatif des investissements directs à l'étranger, passant: de 7,15 mrds d'euros en 2007 à 1,9 mrds d'euros en 2011, et ce, sans que nous assistions en contrepartie à une dynamique du secteur bancaire national occupé par ailleurs comme nous l'avons souligné.

La Roumanie ne semble pas avoir abouti la transition engagée en 1989, et apparaît comme un pays en proie à des vulnérabilités à la fois socio-économiques et territoriales. Son compte de la balance des transactions courantes est négatif $(-23,515$ Mds $€$ en 2008$)^{3}$ et sa dette publique en très forte hausse, passant de $12,6 \%$ du PIB en 2007 à 30,8\% en 2010. Dans ce contexte, on observe une réduction des traitements des fonctionnaires (-25\%), une contraction significative des taux de salaire réels, et une mise à mal du système de protection sociale (Fleuret et Bioteau, 2010). Il en résulte une exclusion financière (monétaire et bancaire) à laquelle est censée répondre la finance solidaire, définit comme l'ensemble des activités d'accompagnement, de financement et de garanties dont l'objet est la consolidation ou la restauration de situations individuelles de plus en plus délicates. En quoi les finances solidaires seraient-elles un outil au service d'un autre développement local dans «une» Roumanie encore en transition?

Pour répondre à cette interrogation, nous retenons un cheminement explicatif en trois temps:

- Pauvreté et finances solidaires: la nécessaire ouverture de l'analyse théorique aux pays d'Europe Centrale et Orientale.

- Regard interdisciplinaire croisé pour mieux comprendre le contexte d'émergence de la finance solidaire en Roumanie.

- Cohésion sociale, dynamique économique locale et finances solidaires: de fortes disparités territoriales en Roumanie.

Nous devrions ainsi à l'issue de cette analyse disposer de premiers éléments approfondis de compréhension des Territoires, territorialités et territorialisations ${ }^{4}$ de la finance solidaire en Roumanie.

3 Notons qu'en 2011, exceptionnellement, cette balance commerciale redevient positive engendrant une croissance économique supérieure à $2 \%$ pour cette année. Mais ce revirement s'explique par des facteurs conjoncturels exogènes, aux premiers rangs desquels nous noterons un hiver 2010-2011 relativement doux (faible consommation de gaz importé) et la crise céréalière globale (la production roumaine, certes moindre que les années précédentes, s'est échangée au prix fort). 4 En référence aux titre et contenus de l'ouvrage éponyme dirigé par Martin Vanier (2009), appliqués à la Roumanie. 


\section{Pauvreté et finances solidaires: la nécessaire ouverture de l'analyse théorique aux pays d'Europe Centrale et Orientale}

Si le système économique des économies centrales et orientales de l'Europe ne peut être appréhendé sans de constantes références à son histoire - ainsi que le fit Seurot (1989, p. 9) pour l'URSS -, il ne peut pas non plus l'être sans que nous consacrions du temps à l'analyse des conséquences sociales des mutations macroéconomiques générées par la perestroïka d'une part, et des conséquences en termes de développement économique local à partir des systèmes de financement locaux d'autre part. En effet, dans le premier cas, il s'agit de comprendre le phénomène de paupérisation induit par cette «restructuration» (Gorbatchev 1987, p. 8) qui, contrairement aux attentes de son auteur, n'a pas permis de «surmonter le processus de stagnation, rompre le mécanisme de freinage, de créer des systèmes fiables et efficaces pour accélérer le progrès social et économique et lui donner une plus grande dynamique»(Gorbatchev, 1987, p. 41). Dans le second cas, en nous référant aux travaux de Samolyk (1994), ${ }^{5}$ nous rappelons que «la santé du secteur financier local peut influencer l'activité d'investissement et la croissance économique régionale en affectant l'aptitude d'une région à financer les projets locaux».

Or, en finances solidaires, telles qu'elles ont été définies par Taupin et Glémain (2007, p. 630), ${ }^{6}$ il s'agit à la fois de palier les creux bancaires induits par les mutations bancaires locales, et de lutter contre les phénomènes de paupérisation en consolidant ou en restaurant des cohésions sociales fragilisées, et des dynamiques économiques locales perdues. Microfinance et développement apparaissent comme des interactions stratégiques triviales dans les «pays» dit «en développement» (PED), il nous semble qu'il n'en est pas encore de même pour les Pays d'Europe Centrale et Orientale en général, même si en Roumanie la microfinance occupe un rôle croissant dans le modèle économique local. Quoiqu'il en soit, en Europe de l'Est, à partir des années 1990 : «en dépit de toutes les différences importantes dans leur histoire, leur culture, et les conditions économiques et politiques, (ses pays) ont en en commun des propriétés importantes, et vont partager des difficultés semblables au cours des années qui viennent» (Kornai, 1990, p. 15). Parmi ces difficultés, on découvre la paupérisation de la Roumanie.

L’approche de la pauvreté en Roumanie proposée par Ionescu (2007) est similaire à celle qui est utilisée en socio-économie et géographie du développement dans les PED. Elle repose sur les six critères suivants:

- Les dimensions de la pauvreté,

- la situation en emploi,

- les conditions d'habitation et d'environnement,

- la structure de la consommation et dotation en équipements électroménagers,

- la santé,

- l'éducation.

$5 \quad$ Cité par Glémain (2000, p. 32).

6 Nous choisissons pour notre part de définir ces finances solidaires comme «un continuum d'activités d'accompagnement et de financement des personnes morales (très petites entreprises) et physiques en difficultés de trésorerie plus ou moins chronique, exclues des services bancaires et financiers de base (bundling)». Cette définition correspond tout autant aux pratiques de microcrédit et de mobilisation (rotative) de l'épargne dans les pays du Sud que de microfinance (microcrédit + microassurance + mobilisation de l'épargne solidaire) dans les pays du Nord. 
Les finances solidaires interviennent traditionnellement, depuis le début des années 1980, dans quatre domaines particuliers du développement local: la création ou la consolidation d'emplois en particulier dans le cadre des petites et très petites entreprises locales, le logement (accès et aménagement), le développement international (aide aux PED), l'accompagnement et le financement du secteur agricole reposant sur de petites exploitations et le secteur de la culture. En l'occurrence, nous retrouvons les affectations «emploi» et « logement» (conditions et équipement) dont les besoins peuvent être couverts par les finances solidaires, ainsi que les dimensions de la cohésion sociale qui se trouvent derrière les domaines inclusifs de la santé et de l'éducation.

En outre, comme le souligne Ionescu (2007, p. 114), la Roumanie fait face à une pauvreté croissante. Ce constat soulève deux questions. La première interroge la qualité territoriale de l'espace concerné par la microfinance: rural ou urbain et, donne ainsi corps territorial au marché initialement envisagé comme un simple lieu théorique d'échange entre l'offre et la demande, aboutissant à un équilibre. La seconde pose celle de l'usage du marché au service d'un projet sociétal (lutte contre les disqualifications socioéconomiques). Elle nous amène ainsi à envisager une lecture Polanyienne du marché de la microfinance en Roumanie selon laquelle: nous serions passés dans les pays en transition comme dans les pays développés d'un modèle où le «marché est au service de la société» à celui d'une «société de marché» validant de facto l'hypothèse TINA «There Is No Alternative», défendue par certains économistes de l'offre (supply side economics) de la Reaganomie et du Thatcherisme. Mais, dans les pays d'Europe Centrale et Orientale comme la Hongrie, il est une forme de tolérance à l'économie privée non lucrative, une sorte d'économie sociale de marché, reposant sur des «vraies» coopératives. ${ }^{7}$ Kornai (1990, p. 93) n'écrit-il pas: «les coopératives pourraient jouer un rôle très utile si les trois principes de base suivants s'y appliquent: tout membre est libre d'y entrer et d'en sortir; quand il en sort, il est libre de reprendre non seulement son capital de départ mais aussi sa part du capital accumulé; la coopérative fonctionne avec un gouvernement propre, librement élu. Une coopérative de ce genre est en fait une forme particulière d'association privée, et n'est donc pas un "grand secteur» indépendant de l'économie, mais une partie du secteur privé?». Pour autant, avons-nous affaire à une économie sociale caractéristique des Pays de l'Est? Le secteur financier local répond-t-il aux besoins de financement de ces acteurs privés «not-for-profit»?

Les années 1990 ont été celles de la privatisation du système bancaire roumain. Au début de l'année 2008, le système bancaire roumain repose sur 29 institutions de crédit de droit roumain, deux caisses d'épargne et de crédit spécialisées dans le secteur du logement locatif, et une coopérative de crédit qui coordonne l'activité de 124 coopératives de crédit, et 13 succursales relevant d'autres institutions de crédit européennes. A ces établissements viennent se greffer des Instituts de Microfinance (IMFs) (Eurom, 2010), plus ou moins difficiles à repérer.

7 Au sujet de la Hongrie, Kornai (1990, p. 93) rejette les «pseudo-coopératives» qui «portent tous les stigmates de la propriété bureaucratique d'Etat. Dans l'idéal ces pseudo-ccopératives devraient se transformer elles-mêmes soit en coopératives authentiques, soit en d'autres unités fonctionnant selon les formes légales ou organisationnelles du secteur privé. Au moins faudrait-il laisser tomber toute prétention, et reconnaître ouvertement que ces pseudo-coopératives sont propriétés d'Etat. En tout cas l'économie hongroise devra finir par s'en débarrasser». 


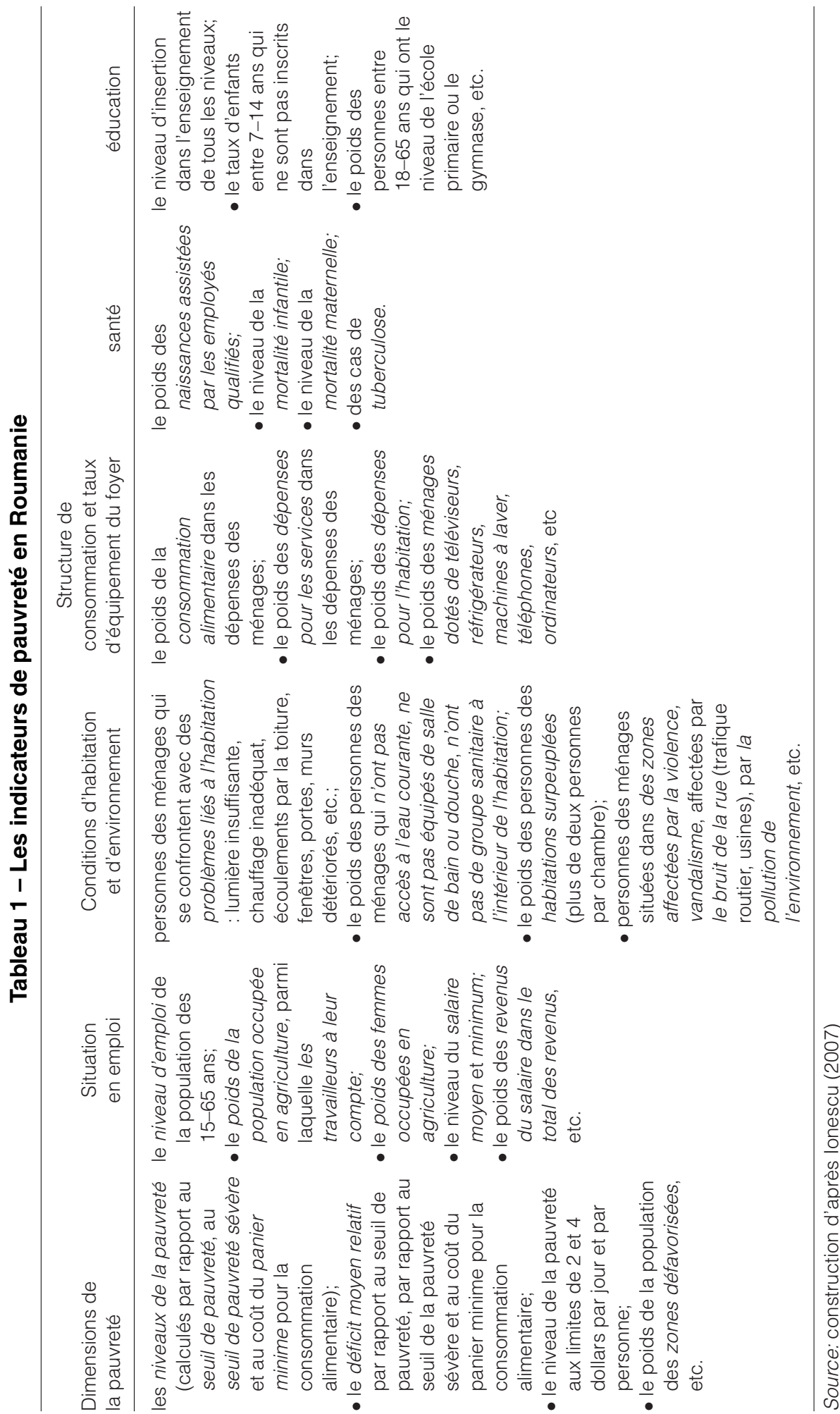


Tableau 2 - Les économies de la grandeur pour comprendre le système bancaire et financier roumain

\begin{tabular}{|c|c|c|c|c|}
\hline Monde & $\begin{array}{l}\text { Grandeurs (base } \\
\text { des conventions) }\end{array}$ & Espace & Relations & Acteur roumain \\
\hline Industriel & $\begin{array}{l}\text { Efficacité économique } \\
\text { (massification, } \\
\text { standardisation) }\end{array}$ & $\begin{array}{l}\text { Le national et } \\
\text { l'Européen }\end{array}$ & $\begin{array}{l}\text { Fonctionnelles et } \\
\text { anonymes }\end{array}$ & $\begin{array}{l}\text { Système bancaire roumain } \\
\text { «privatisé» }\end{array}$ \\
\hline Marchand & $\begin{array}{l}\text { Profit personnel } \\
\text { (résultat } \\
\text { économique, taille) }\end{array}$ & $\begin{array}{l}\text { «sans» i.e. partout } \\
\text { dans le cadre } \\
\text { mondialisé }\end{array}$ & Opportunistes & $\begin{array}{l}\text { Instituts de microfinance } \\
\text { «commerciauX» (Social } \\
\text { Business): CAPA } \\
\text { finance, ROMCOM, } \\
\text { CDE, UNCAR }\end{array}$ \\
\hline Civique & $\begin{array}{l}\text { Solidarité (intérêt } \\
\text { collectif, } \\
\text { soutenabilité sociale) }\end{array}$ & $\begin{array}{l}\text { Englobant et } \\
\text { englobé } \\
\text { (construit social), } \\
\text { le local }\end{array}$ & $\begin{array}{l}\text { Egalitaires, de soutien } \\
\text { (accompagnement } \\
\text { et suivi) }\end{array}$ & $\begin{array}{l}\text { Instituts de microfinance } \\
\text { de proximité (sociale et } \\
\text { géographique) (Social } \\
\text { Economy): LAM, OMRO, } \\
\text { Express Finance, } \\
\text { INTEGRA, FAER }\end{array}$ \\
\hline
\end{tabular}

Dans l'absolu, il nous semble pourtant possible de mobiliser l'économie des grandeurs de Boltanski et Thévenot (1991) pour comprendre la biodiversité contemporaine du système bancaire et financier roumain. En effet, eu égard à ce que nous y observons, nous posons l'hypothèse d'une coexistence de trois mondes le composant: le monde industriel, le monde marchand et, le monde civique, que nous synthétisons dans le tableau ci-après (Tableau 2).

A la lecture de ce tableau de synthèse, qui repose sur l'hypothèse d'une grille de lecture des économies de la grandeur, il apparaît que l'appréhension et la représentation de l'espace différent selon les mondes et, donc selon les acteurs qu'ils relèvent du Social Business ou bien de la Social Economy. Quels sont les apports de la géographie économique et sociale à notre compréhension?

\section{Regard interdisciplinaire croisé pour mieux comprendre le contexte d'émergence de la finance solidaire en Roumanie}

Au regard de la situation délicate de la Roumanie aux niveaux à la fois économique et social que nous avons déjà dévoilée, on peut à juste titre s'interroger sur le niveau de transition de ce pays, ainsi que sur sa capacité à porter des innovations financières situées, ${ }^{8}$ c'est-à-dire: établissant un pont entre les individus et la société en voie de "marchéisation», dans laquelle ils évoluent. Pour apporter des éléments de réponse à cette interrogation, nous allons procéder en deux temps. Après avoir conduit une analyse économique des systèmes de financement locaux en transition et en émergence avec le secteur de la microfinance, ${ }^{9}$ nous proposerons

8 H. Zaoual (2004), définit ainsi le site.

9 Nous comprenons par microfinance, l'ensemble des activités de microcrédits, de microassurance et de mobilisation de l'épargne solidaire. Le caractère solidaire de l'épargne signifie qu'elle 
une analyse géographique des déterminants territoriaux de la finance solidaire ${ }^{10}$ en Roumanie.

Partant de l'hypothèse d'une économie des régions en Europe, ${ }^{11}$ nous cherchons à participer à la compréhension des dynamiques économiques territoriales portées par des réseaux ou des acteurs nouveaux du financement des économies et des sociétés locales, selon un modèle d'économie de proximités (sociale et géographique), au service d'un développement socialement soutenable et situé.

2.1 Analyse économique des systèmes de financement locaux en transition et en émergence

A la fin des années 1990, faisant suite à la demande d'adhésion à l'Union européenne de 10 Pays d'Europe Centrale et Orientale (PECO) entre 1994 et 1996, la Commission européenne a entrepris une évaluation des capacités des différents Etats centre européens à respecter les critères discriminants de l'Agenda 2000 (Commission européenne, 1997). Seules l'Estonie, la Hongrie, la Pologne, la République Tchèque et la Slovaquie les respectaient alors simultanément:

- L'existence d'une économie de marché viable, la capacité à faire face aux pressions concurrentielles et aux forces du marché au sein de l'UE,

- La garantie de la démocratie avec des institutions stables, la primauté du droit, le respect des droits de l'Homme, le respect des minorités et leur protection,

- La capacité à assumer les obligations de l'adhésion, soit souscrire aux objectifs de l'Union politique, économique et monétaire.

A l'instar de la Bulgarie, de la Lettonie et de la Lituanie, la Roumanie ne respectait ni le premier, ni le troisième critère. Le tableau suivant (Tableau 3) rend compte des atouts et des difficultés initiales, de quelques PECO et de l'URSS pour faire face à la problématique de la transition. La Roumanie est, de tous, le moins bien classé à l'époque - ce classement se confirme aujourd'hui encore: les PNB de ces différents Etats les positionnent actuellement dans le même ordre de classement (Banque Mondiale).

En 2008, faisant suite à l'adhésion l'année précédente de la Roumanie et de la Bulgarie à l'Union européenne, la Commission européenne procède à une nouvelle étude qui, à ce moment, pointe la persistance de difficultés structurelles, telles que: le manque de préparation des ressources humaines au modèle d'économie de marché, les défauts de perception de la TVA et des suivis des flux financiers. Ce dernier point d'alerte interroge directement le système bancaire roumain.

Au-delà de ces conditions communautaires, ce sont implicitement les capacités disponibles de financement du pays, ainsi que les éléments de cohésion sociale qui lui

a vocation à consolider ou à créer des emplois, des activités nouvelles génératrices de revenus ou d'utilité sociale.

10 La microfinance à laquelle on ajoute les activités de garanties (couverture de risques de défaut).

11 Nous acceptons ici l'hypothèse d'une Politique Européenne d'Aménagement du Territoire telle qu'elle est prévue dans le programme «Europe 2000 +» (Du Granrut 1996, p. 146, et Glémain (2000)). 
Tableau 3 - Atouts initiaux des «Pays de l'Est» face à la transition

\begin{tabular}{lccccc}
\hline Pays & $\begin{array}{c}\text { Degré de conviction } \\
\text { vis-à-vis du marché }\end{array}$ & $\begin{array}{c}\text { Capacité à adopter } \\
\text { des comportements } \\
\text { marchands }\end{array}$ & $\begin{array}{c}\text { Critères } \\
\text { économiques } \\
\text { internes }\end{array}$ & $\begin{array}{c}\text { Critères } \\
\text { économiques } \\
\text { externes }\end{array}$ & Total \\
\hline URSS & 6 & 6 & 7 & 2 & 6 \\
Bulgarie & 5 & 3 & 1 & 5 & 5 \\
Hongrie & 1 & 2 & 4 & 7 & 3 \\
Pologne & 2 & 1 & 5 & 4 & 2 \\
Roumanie & $\mathbf{7}$ & 7 & $\mathbf{6}$ & $\mathbf{1}$ & $\mathbf{6}$ \\
Tchécoslovaquie & 4 & 3 & 2 & 3 & 1 \\
RDA & 2 & 5 & 3 & 6 & 4 \\
\hline
\end{tabular}

Lecture: classement des pays selon leurs atouts: $1=$ premier rang.

Source: Tiraspolsky, Wild (1992) cité par Ricoeur-Nicolaï et al. (1999, p. 1113)

sont inhérentes (accès à l'argent et aux services bancaires de base) qui sont ignorées. Or, cette fonction qui combine financement du développement économique local et consolidation de la cohésion sociale locale, caractérise la finance solidaire, donc les activités de microfinance et de garanties des fonds mobilisés au service de ces territoires.

\subsection{En Roumanie: un système bancaire domestique encore en transition?}

Depuis les années 1980, les systèmes bancaires en Europe ont subi les effets conjugués de la déréglementation, de la déspécialisation, et de la libéralisation. Il en résulte l'instauration d'une industrie bancaire qui rend les établissements bancaires isomorphes, c'est-à-dire de moins en moins distincts les uns des autres. Par exemple, les établissements bancaires coopératifs seraient de moins en moins différents des établissements bancaires commerciaux. La question est de savoir si nous n'assisterons pas à un «retour sur place» avec une biodiversité bancaire en Europe, telle qu'elle a été analysée par Glémain (2000)? Qu'en est-il du système bancaire roumain?

En 1990, la Roumanie crée la Banque Commerciale de Roumanie (BCR) pour porter les opérations commerciales de la Banque Nationale de Roumanie (BNR). Cette tendance à la commercialisation des opérations des banques est renforcée par la Loi Bancaire ${ }^{\circ} 58 / 1998$ qui privatise d'une part la Banque Roumaine de Développement dont le capital s'ouvre à hauteur de $41 \%$ à la Société Générale (135 millions d'USD) et, d'autre part, la Banc Post (BP) dont le capital est détenu depuis à 45\% par la General Electric Capital et la Banco Portuguese de Investimento. En 2001, c'est la Banque Agricole qui devient Raiffeissen Bank SA. Ce sont ainsi 45 établissements bancaires qui animent le système roumain en 2008, contre seulement 11 en 1990 . Pour autant, à la lecture des tableaux 3 et 4 , on constate qu'un oligopole de 3 établissements bancaires porte plus de $50 \%$ des parts de marché. De fait, le nombre de banques pour 100.000 habitants plafonne à 0,18 .

Le paysage bancaire roumain est donc relativement concentré avec des établissements bancaires pour l'essentiel «commerciaux». Il est ainsi d'abord tourné vers le financement des grands secteurs économiques nationaux aux dépens du financement des petites activités industrielles et commerciales à l'échelle locale. De 


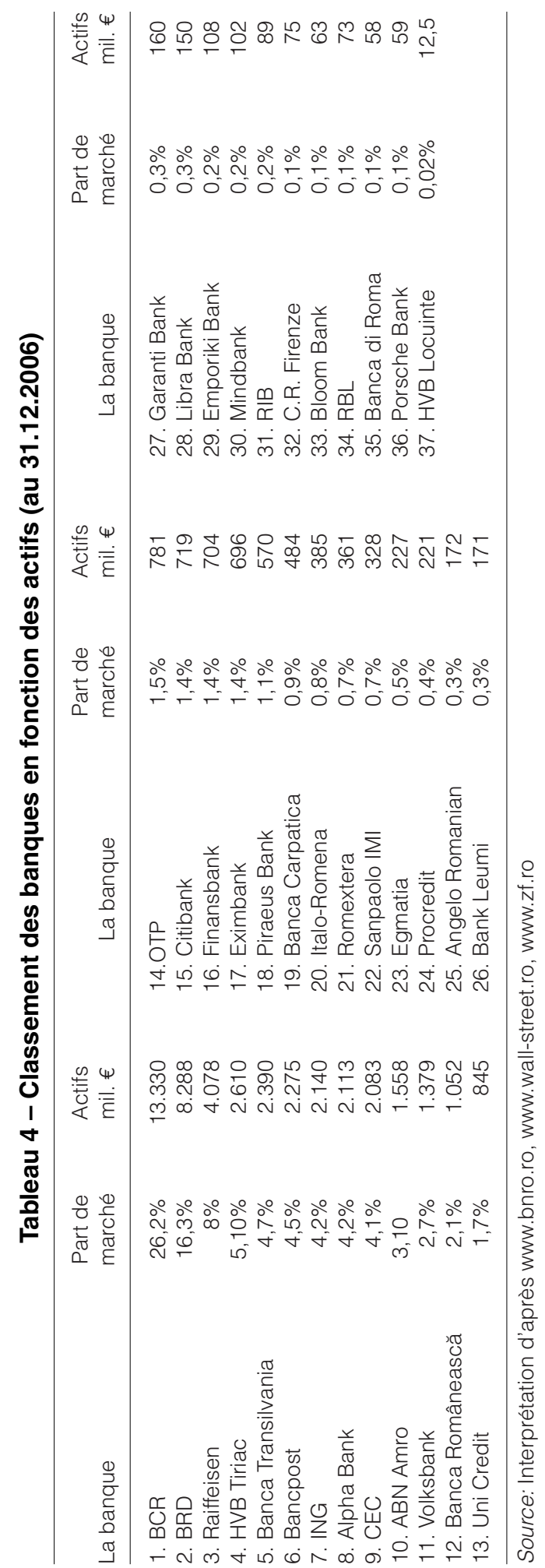


Tableau 5 - Evolution de la part de marché des 10 premières banques commerciales de Roumanie entre 2003 et 2006

\begin{tabular}{|c|c|c|c|c|c|c|c|}
\hline \multicolumn{3}{|c|}{ Rang } & \multirow[b]{2}{*}{ La Banque } & \multirow[b]{2}{*}{2003 (\%) } & \multirow[b]{2}{*}{2004 (\%) } & \multirow[b]{2}{*}{2005 (\%) } & \multirow[b]{2}{*}{2006 (\%) } \\
\hline 2006 & 2005 & 2004 & & & & & \\
\hline 1 & 1 & 1 & 1. $B C R$ & 29,3 & 26,1 & 25,7 & 26,2 \\
\hline 2 & 2 & 2 & 2. BRD & 13,3 & 13,0 & 15,0 & 16,3 \\
\hline 3 & 3 & 3 & 3. Raiffeisen & 6,9 & 9,2 & 8,7 & 8,0 \\
\hline 4 & 5 & 7 & 4. HVB Tiriac & 5,9 & 7,3 & 7,4 & 5,1 \\
\hline 5 & 8 & 10 & 5. Banca Transilvania & 2,3 & 2,9 & 3,9 & 4,7 \\
\hline 6 & 6 & 8 & 6. Bancpost & 4,1 & 4,6 & 4,5 & 4,5 \\
\hline 7 & 4 & 5 & 7. ING & 4,2 & 5,6 & 5,3 & 4,2 \\
\hline 8 & 9 & 9 & 8. Alpha Bank & 3,3 & 3,2 & 3,8 & 4,2 \\
\hline 9 & 7 & 4 & 9. CEC & 6,9 & 5,9 & 4,4 & 4,1 \\
\hline 10 & 10 & 6 & 10. ABN Amro & 5,1 & 4,9 & 3,7 & 3,1 \\
\hline
\end{tabular}

Source: Interprétation d'après www.bnro.ro, www.wall-street.ro, www.zf.ro

Tableau 6 - Une comparaison de l'approche mécaniste et de l'approche interactive

Rationalité mécaniste

(individualisme méthodologique)

Rationalité interactive

Un acteur confronté à l'environnement

Recours à l'utilisation de probabilités subjectives pour se ramener à un modèle complet de l'environnement.

Le moteur du processus de décisions: I'incitation individuelle.

L'approche est positive: tout se passe «comme s'il suffit d'appliquer la solution».

Le facteur clé de succès: le modèle est robuste et constitue une bonne approximation de la réalité.
Plusieurs acteurs en interaction

Recours aux spécificités objectives de la situation pour réduire en partie l'incertitude éventuellement au prix de conventions et d'arbitraire.

Le moteur de décision: la recherche de point focal.

L'approche est constructiviste: le modèle doit être constamment dépassé, il n'est qu'une référence pour l'action.

Le facteur clé de succès: une procédure d'invalidation particulièrement discriminante.

Source: Ponsard (2004, p. 206)

la sorte, nous avons affaire à un creux bancaire qui, depuis 2008, est comblé, pour partie, par les coopératives de crédit et, pour l'autre, par les institutions de microfinance. Cela explique dans une certaine mesure le fait que la Roumanie apparaisse comme le second pays en termes d'activité de microcrédit en Union européenne.

Contrairement à l'action publique (Gilbert, 1996, p. 311), l'action bancaire ne s'inscrit pas en Europe dans «les limites d'un territoire au-delà desquelles les autorités ne disposent pas du support juridique nécessaire. En ce sens, le cadre pertinent pour l'économie publique est un cadre spatialisé», alors que celui de l'économie bancaire est un cadre territorialisé. Dès lors, nous serions plutôt en présence d'une rationalité interactive distincte de l'approche mécaniste (Tableau 6).

Sous cette hypothèse, la microéconomie bancaire territorialisée de la Roumanie ouvre des perspectives d'interaction entre les acteurs présents, bien que l'on puisse envisager que les plus petits disparaissent ou bien se regroupent. Mais, la décision consiste bien en une recherche d'un point focal que le système bancaire roumain semble avoir perdu. Le secteur de la microfinance peut-il l'aider? 
Tableau 7 - Evolution du nombre de Petites et Moyennes Entreprises en Roumanie

\begin{tabular}{lrrrrrrrrrr}
\hline Taille & 2000 & 2001 & 2002 & 2003 & 2004 & 2005 & 2006 & 2007 & 2008 & 2009 \\
\hline Micro & 375804 & 374255 & 377499 & 417366 & 358787 & 386561 & 410759 & 431021 & 602711 & 573299 \\
Petites & 29121 & 30340 & 30231 & 33856 & 36392 & 39128 & 43419 & 47022 & 49560 & 43724 \\
Moyennes & 7504 & 7737 & 7761 & 8147 & 9121 & 9158 & 9322 & 9577 & 9753 & 8435 \\
TOTAL & 412429 & 412332 & 415491 & 459369 & 404300 & 434847 & 463500 & 487620 & 662024 & 626458
\end{tabular}

Sources: Etude d'impact de la crise dans le secteur des PME : http://www.revecon.ro/articles/2011-2/ 2011-2-5.pdf; INSSE; MEBE; ONRC

\subsection{Un secteur de la microfinance émergent en Roumanie}

Le secteur de la microfinance en Roumanie se développe depuis le début des années 1990. Il s'inscrit dans une forte croissance économique qui a ouvert des opportunités de créations de petites et micro-entreprises comme dans le reste de l'Europe. Mais, les micro-entreprises représentent à elles-seules environ $90 \%$ du total des entreprises du pays en activité (Tableau 7).

Or, à l'instar de ce que nous observons en France (Glémain, Bioteau, Ribeiro, 2012), ces micro-entreprises (encadré 1.) doivent être appréhendées comme autant d'initiatives entrepreneuriales individuelles solidaires, qui «répondent à des attentes locales (rurales ou urbaines) nouvelles ou renouvelées qui correspondent à de micro besoins» (Manoury 2001, p. 47).

\section{Encadré 1. De la définition des «PE» et «ME» dans l'Union européenne.}

JO de l'UE 20-5-2003 2003/361/CE C(2003)1422 L124/39.

Dans la catégorie des petites et moyennes entreprises (PME), l'Union européenne distingue:

- les petites entreprises (PE) de moins de 50 personnes et, dont le chiffre d'affaires ou le total du bilan n'excède pas 10 millions d'euros;

- les micro-entreprises (ME) de moins de 10 personnes et, dont le chiffre d'affaires ou le total du bilan n'excède pas 2 millions d'euros.

Ces micro-entreprises sont en besoin de financement pour l'investissement, auquel ne répondent pas les établissements bancaires nationaux. En revanche, depuis la loi 25/2006, la Roumanie reconnaît par enregistrement et octroi de licence les Instituts de Microfinance comme de nouveaux acteurs du financement du développement économique local et, de la cohésion sociale car ils créent des emplois et, répondent à des communautés spécifiques telles que: les Roms. Neuf IMF animent pour l'essentiel le paysage roumain de la microfinance (Tableaux 8a. et 8b.).

Ces premiers Instituts de Microfinance (IMF) présentent la caractéristique d'accompagner à la fois la dynamique des tissus productifs locaux en soutenant la création ou l'expansion des petites et micro-entreprises (PEME), en luttant ainsi contre le chômage, et le développement territorial, en ayant le souci de soutenir les espaces ruraux. C'est donc une microfinance à la fois sociale et territoriale, répondant du modèle que nous appellerons de "proximité géographique et d'innovation». 
Tableau 8a - Les IMFs roumains territorialisés

\begin{tabular}{|c|c|c|c|}
\hline & Express Finance & $\begin{array}{l}\text { Opportunity Microfinance } \\
\text { Romania (OMRO) }\end{array}$ & $\begin{array}{c}\text { INTEGRA microfinance } \\
\text { IFNSA }\end{array}$ \\
\hline Année de création & 1996 & 1995 & 2000 \\
\hline Localisation & Timisoara & Régions centrales & Oradea, Brasov \\
\hline Inspiration & $\begin{array}{l}\text { Model Project for World } \\
\text { Dévelopment }\end{array}$ & Fondation Izvor & $\begin{array}{l}\text { Economie communautaire } \\
\text { des Pays de l'Est }\end{array}$ \\
\hline $\begin{array}{l}\text { Portefeuille de prêt brut } \\
\text { en euros }\end{array}$ & 8,3 millions & 9,7 millions & 313222 \\
\hline $\begin{array}{l}\text { Nombre d'emprunteurs } \\
\text { Actifs }\end{array}$ & 2366 & 2155 & 135 \\
\hline Objet & $\begin{array}{l}\text { Services aux personnes } \\
\text { en zone urbaine }\end{array}$ & $\begin{array}{l}\text { Soutien à la création d'emplois } \\
\text { dans l'agriculture, le } \\
\text { commerce, les services }\end{array}$ & $\begin{array}{l}\text { Micro-entreprises, } \\
\text { entrepreneuriat en milieu } \\
\text { rural, entrepreneuriat au } \\
\text { féminin }\end{array}$ \\
\hline
\end{tabular}

Tableau 8b - Les IMFs roumains territorialisés

\begin{tabular}{|c|c|c|}
\hline & LAM & $\begin{array}{l}\text { Foundation for the Promotion of } \\
\text { Agricultural and Regional Economy (FAER) }\end{array}$ \\
\hline Année de création & 1992 & 1992 \\
\hline Localisation & Covasna, Harghita, Brasov & Maramures, Bistrita,Suceava \\
\hline Inspiration & $\begin{array}{l}\text { Organisation pour aider les Eglises } \\
\text { encycliques en } \\
\text { Suisse-Conféderation Suisse }\end{array}$ & $\begin{array}{l}\text { Organisation pour aider les Eglises } \\
\text { encycliques en Suisse-Confédération Suisse }\end{array}$ \\
\hline $\begin{array}{l}\text { Portefeuille de prêt brut } \\
\text { en euros }\end{array}$ & 2,42 millions & 1,43 million \\
\hline $\begin{array}{l}\text { Nombre d'emprunteurs } \\
\text { Actifs }\end{array}$ & 698 & 609 \\
\hline Objet & $\begin{array}{l}\text { Soutien au développement du } \\
\text { secteur agricole privé, et, soutien } \\
\text { à l'installation de petites et } \\
\text { micro-entreprises en secteur rural }\end{array}$ & $\begin{array}{l}\text { Aide à l'installation d'exploitations agricoles et } \\
\text { de PME en milieu rural, soutien au tourisme } \\
\text { rural, aide à l'accès au financement là où il } \\
\text { n'existe pas de système bancaire local }\end{array}$ \\
\hline
\end{tabular}

Les quatre autres acteurs de la microfinance en Roumanie se caractérisent ainsi :

Ce deuxième groupe d'IMFs illustre le volet «commercial» de la microfinance en Roumanie, ainsi que son inspiration du modèle anglo-saxon de l'entrepreneuriat social. En effet, il promeut l'accompagnement et le financement d'entreprises de capitaux à objet social. Il rejoint à ce titre le modèle du capitalisme moral de M.Yunus (2008). Ce sont des «entreprises qui gagnent de l'argent sans être exclusivement tendue vers la maximisation du profit et, qui consacre leurs bénéfices à la diminution des coûts et, à la production d'avantages sociaux sans que les actionnaires ne soient rémunérés». Il s'agit dès lors d'un modèle économique de justice à la John Rawls.

\section{Cohésion sociale, dynamique économique locale et finances solidaires: de fortes disparités territoriales en Roumanie}

La section qui précède rend compte de problématiques spécifiquement adossées aux transformations / mutations du système bancaire et à l'émergence de la 


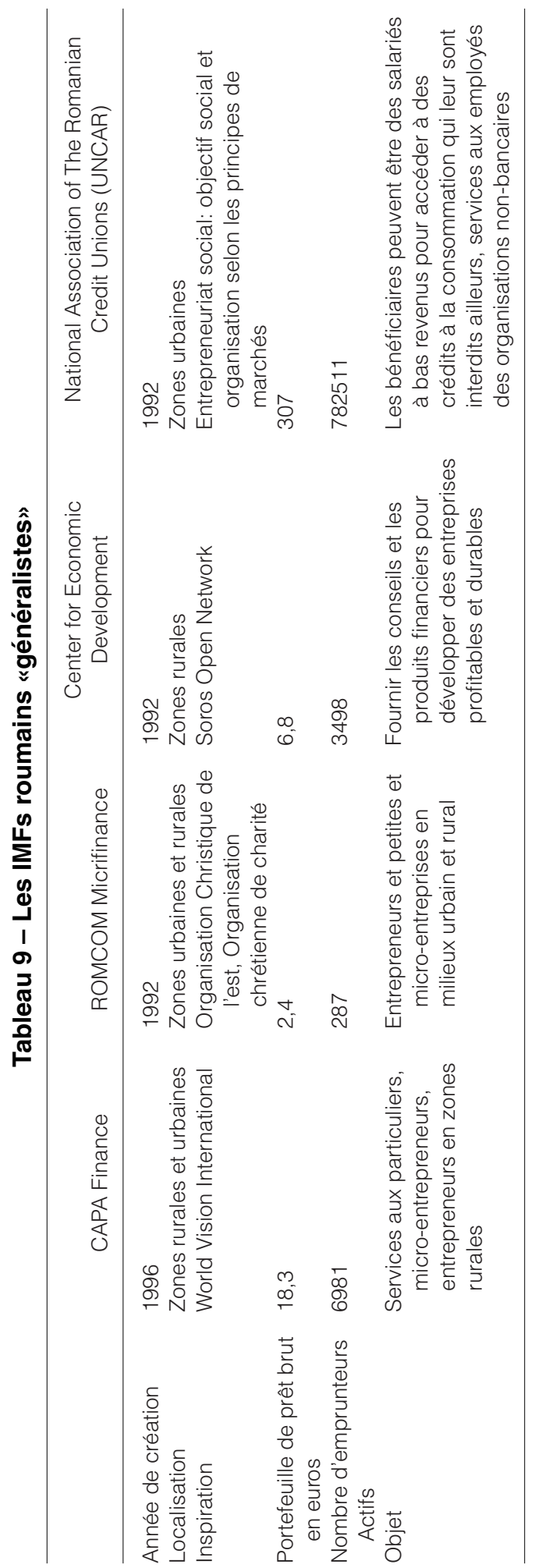


microfinance. Le contexte général, tel que nous le constatons, est celui d'une économie (encore) en transition, qui peine en particulier à faire face aux besoins civiques décrits au travers de l'approche par les économies de la grandeur. Ce contexte interroge les outils à disposition d'un Etat encore faiblement décentralisé, dans lequel les régions sont faiblement développées comparativement aux moyennes européennes (l'ensemble du pays est éligible au Fond de cohésion, représentant moins de $70 \%$ de la moyenne de PIB européen).

Surtout, les perspectives d'investissement en infrastructures (Corridor de transport européen numéro IV - autoroutier et ferroviaire - reliant la Mer Noire à la Hongrie via Bucarest et les villes de Transylvanie) ou dans le secteur tertiaire, favorisent globalement les grandes villes, et font que les chances de réduire les écarts sont encore moindre à courts et moyens termes. Au-delà du seul contexte socioéconomique territorialisé, la géographie économique et sociale peut, par le prisme qu'elle propose, en considérant les dimensions spatiales de la société, permettre de mieux appréhender les problématiques et enjeux auxquels la microfinance doit faire face. Au final, cette démarche conduit à définir une typologie des territoires (les construits dans l'espace), des territorialités (les problématiques et enjeux auxquels faire face), jusqu'à la territorialisation qui en découle (les choix et mode de construction des territoires) de la finance solidaire en Roumanie. Cette approche, pour originale qu'elle est, permet de combiner différents regards et différentes perspectives sur la finance solidaire, entre enjeux globaux et dynamiques locales desquels participe, et conjointement, la microfinance.

\subsection{Roumanie. Comprendre l'origine des disparités socioéconomiques territorialisées}

La Roumanie est organisée depuis 1968 en 41 départements (judeţ) auxquels s'ajoute la municipalité de Bucarest. La superficie moyenne est ainsi de 5900 $\mathrm{km}^{2}$ /département et la population moyenne de 500.000 hab./département (INSSE, ${ }^{12}$ 2012). Le pays a amorcé dans les années 1990 une régionalisation, non pas administrative mais technique: pour planification du développement et pour gestion de fonds, notamment des fonds européens. Les régions de développement sont au nombre de 8 . Elles regroupent les 41 départements et la municipalité de Bucarest dans des territoires d'une taille moyenne de $33000 \mathrm{~km}^{2}$ (INSSE, 2012).

Cette construction fait émerger un nouveau palier territorial «sans pouvoir et sans territoire» (Boulineau, Suciu, 1996), car la capacité de décision en termes d'aménagement et de déploiement des politiques publiques reste départementale. Il n'est pas encore de réelle cohérence à dimension régionale en Roumanie. Ces régions sans personnalité juridique ne sont pas appropriées par les habitants.

Malgré ses tentatives politiques réitérées de réduction des disparités régionales (en période communiste, et depuis lors avec la régionalisation), la Roumanie connaît toujours, et de plus en plus, de forts contrastes de développement entre rural et urbain, Ouest et Est, et même infrarégionaux. La régionalisation a été conçue dans l'objectif d'associer des départements en difficultés économiques, démographiques... aux départements jugés plus dynamiques. Mais les réalités sociospatiales interdisent

12 INSSE: Institut National de la Statistique et des Etudes Economiques de Roumanie. 
de corriger à l'échelle régionale des écarts qui, dans les faits, doivent être considérés au niveau du territoire étatique.

Le semis des villes est à peu près régulier en Roumanie (Rey et al., 2007, p. 74), dû notamment à l'effort d'urbanisation de la période communiste. Mais des écarts régionaux relativement fins peuvent être décelés. Ainsi, on constate une urbanisation plus prégnante dans l'ouest du pays, au contraire des régions du sud: notamment dans les 4 départements voisins de Bucarest, dont le taux d'urbanisation n'excède pas $40 \% .^{13}$ Or, les principales villes constituent également les principaux pôles de croissance en Roumanie: les régions les plus urbanisées sont également celles dans lesquelles la croissance économique a été la plus vive avant crise (de 2008) (d'après données INSSE).

Les départements de Constanta (port maritime), Galati (sidérurgie) et Arges (industrie automobile), ainsi que la ville de Bucarest et le département d'Ilfov qui la ceinture semblent des cas isolés de pôles de croissance dans le Sud et l'Est du pays. Sans eux il se dessine une cartographie distinguant la Transylvanie (à l'intérieur de la courbure des Carpates et à l'ouest) du reste du territoire roumain. Les contreforts frontaliers de la République de Moldavie, de l'Ukraine, du bassin de la Mer Noire et de la Bulgarie comme de la Serbie sont peu attractifs pour l'investissement étranger.

Enfin, corrélée avec des éléments de positionnement spatial comme la proximité d'une frontière d'Etats, en particulier la Hongrie et la Mer Noire, ou de très grandes villes (Bucarest, et dans une moindre mesure Timisoara, Cluj, Constanta, Galati-Braila), ou en lien avec des évènements conjoncturels comme la disponibilité immédiate de bâtiments suite à des restructurations industrielles, la présence d'une main d'oeuvre plus nombreuse et qualifiée dans les départements à dominante urbaine a favorisé les investissements étrangers. Si pour les départements urbanisés de Transylvanie, le potentiel induit par la position était un facteur important pour les investisseurs étrangers, les régions plus éloignées et plus rurales comme le Nord-est, le Sud-est ou le Sud-ouest connaissent un volume d'investissement moindre, de moins de $100 € /$ hab. entre 1991 et 2005 !

Ainsi nous faisons face à une économie en transition dans laquelle l'essentiel des investissements joue d'une rente de situation: au plus proche des espaces d'exportation, en direction de l'ouest européen pour l'heure. Le tissu urbain favorise le déploiement de la microfinance, tandis que l'émergence des activités de service et d'artisanat en milieu rural s'accompagne de besoins de financements auxquels la microfinance peut répondre. En revanche, les profils d'activités en milieu rural ne favorisent pas l'investissement: productions agricoles de subsistance, artisanat peu mécanisé et peu renouvelé dans ses pratiques. Ces activités constituent globalement par leurs profils un frein à la microfinance alors que celle-ci pourrait être une solution pour nombre de familles.

\subsection{Une géographie économique et sociale de la microfinance en Roumanie}

Les principales villes concentrent les sièges des principales institutions financières (banques, assurances) et des organisations de soutien à l'activité économique. Elles

13 Cette caractéristique régionale s'explique à proximité de Bucarest par l'attractivité de cette dernière et du département la ceinturant (Ilfov) qui bien qu'encore peu urbanisé accueille de plus en plus de périurbains. 
accueillent également des institutions de formation de type universitaire. Ainsi, la donnée relative à l'urbanisation renvoie en Roumanie à des critères d'équipements, d'accès aux services, de potentiels fiscaux des collectivités, ou encore de densité du tissu d'établissements autres que de production agricole. Dans les départements à dominante urbaine, l'activité villageoise non agricole se déploie autour de commerces et services de proximité ainsi que par le développement d'un petit artisanat, voire parfois de petites industries en quête de main-d'œuvre. Ce phénomène caractérise en particulier l'ouest du pays. ${ }^{14}$

A l'opposé, les départements à dominante rurale (il subsiste des départements dans lesquels plus d'1/3 des actifs sont agriculteurs !) sont marqués par une (encore) forte fréquence d'exploitations agricoles vivrières ou semi-vivrières dont l'essentiel des ventes est réalisé sur les marchés locaux, villageois. Ces exploitations sans capitaux, réalisent peu voire pas d'investissements. Les rares industries ou services spécialisés sont cantonnés aux villes, enregistrées en faible nombre.

Ce qui pourrait constituer un troisième «rythme» est l'ensemble des départements à forts potentiels touristiques (montagnes: Carpates ou Apuseni; patrimoine: régions à fortes densités de monastères; littoral... ). L'agriculture y maintient des paysages et des modes de productions traditionnels. ${ }^{15}$ De plus en plus fréquemment dans ces contextes, l'activité agricole se double d'une activité en tourisme-hôtellerie. Ces campagnes développent enfin des activités d'artisanat (et de commerce) à destination de cette clientèle touristique.

Sur la base de ces différents constats, nous pouvons alors dresser un portrait de territoire de la Roumanie à même de renseigner les potentiels de développement de la microfinance. Ce potentiel est inégal en fonction des profils territoriaux identifiés. Il nous conduit à définir une typologie des territoires de la microfinance qui, à l'instar de ce qui peut être observé concernant les profils économiques départementaux, dissocie régions à dominante urbaine et régions plus rurales d'une part, ouest et est du pays d'autre part.

Les organismes de microcrédit sont plus densément présents au sein des deux régions de développement Nord-ouest et Centre (Carte 1), marquées conjointement par la forte présence de la minorité magyare, par une tradition de l'échange dans l'Europe germanique et, sans lien de cause à effet, par une forte densité de présence des cultes dits néo-protestants (Baptistes, Pentecôtistes, Adventistes ... ). Différents travaux (Bioteau, 2005; Capelle-Pogacean, Michel et Pace, 2008; Capelle-Pogacean et Ragaru, 2008) montrent les liens entre minorités magyares, présence allemande massive par le passé mais toujours maintenue et le développement de relations inter-ONG entre Roumanie, Hongrie et surtout Allemagne. Pour ce qui concerne le néo-protestantisme, ces mêmes travaux mettent à jour l'existence de réseaux confessionnels internationaux à même de proposer des solutions de financement de l'initiative entrepreneuriale.

14 L'essentiel de l'activité agricole est aujourd'hui le fait de grandes exploitations qui soit prolongent l'activité des sociétés agricoles d'Etat ou des coopératives de la période communiste, soit se sont refondées à l'appui, le plus souvent, d'investissements étrangers.

15 Précisons que dans ces mêmes départements, la collectivisation des terres n'a été que partielle. Cette continuité de la propriété foncière a eu pour effets le maintien de modes de production mixtes: agricoles et artisanaux à la fois, et la préservation du bâti traditionnel à fort potentiel paysagers et touristique. 


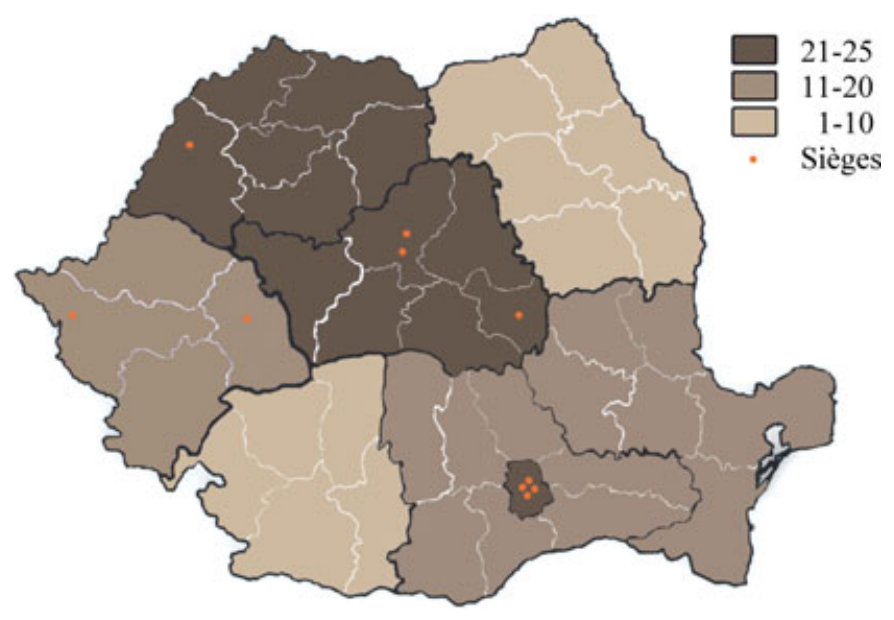

Figure 1 - Densités régionales de l'offre (opérateurs de microcrédit intervenant dans le pays) et sièges des organismes de microcrédit en Roumanie en 2009 (d'après EUROM).

Nous sommes confrontés à une géographie qui tend à confirmer une typologie suivant laquelle l'offre de microcrédit en Roumanie est révélatrice:

- Des potentiels à investir de la population. Elle est peu présente dans les régions où domine l'agriculture vivrière, de quasi subsistance, confirmant que la microfinance roumaine est une microfinance d'un Etat déjà avancé sur le plan économique (du Nord), qui ne s'adresse pas aux populations les plus démunies mais à des exclus ou à des isolés bancaires (Glémain et al., 2010).

- Des héritages historiques ou tout au moins des liens communautaires. Les régions à forte présence de minorités sont aussi celles dans lesquelles l'offre de microcrédit est la plus diverse. Certains réseaux confessionnels notamment déploient leurs propres offres.

- Du potentiel économique intrinsèque des régions. L'ouest proche des frontières d'exportation, Bucarest concentrant l'essentiel des richesses et le pouvoir politique, le littoral de la Mer Noire... sont autant de régions dans lesquelles l'investissement jouit de facteurs favorables. Les organismes de microfinance y sont présents plus massivement.

\section{Conclusion. Territoires, territorialités et territorialisations ${ }^{16}$ de la finance solidaire en Roumanie}

Le concept de territoire est assez récent dans la géographie comme dans l'ensemble des sciences de l'Homme ainsi qu'en sciences économiques. Au-delà du débat sur sa polysémie fréquemment dénoncée (du territoire administratif ou du territoire socialement construit) succèdent aujourd'hui des travaux qui, en géographie, considèrent par le territoire, ses formes, son animation plurielle, ses acteurs, ... l'expression de la (des)

16 En référence aux titre et contenu de l'ouvrage éponyme dirigé par Martin Vanier (2009). 
dimension(s) spatiale(s) des sociétés (Séchet et Keerle, 2009). Le territoire est ainsi le reflet des projets sociétaux et se trouve en permanence redéfini par les choix de société.

L'offre de microcrédit participe de cette dynamique. Les organismes porteurs de microcrédits définissent leurs propres territorialités, c'est-à-dire leurs propres approches $\mathrm{du}$ territoire en fonction des enjeux qu'ils identifient dans le territoire. Il s'ensuit une territorialisation spécifique de leur activité. Nous l'avons vu en première section de ce texte (tableaux 7a, 7b et 8): les territorialisations de leurs activités diffèrent et rélèvent, comme elles révèlent des choix de politique institutionnelle, de la diffusion du microcrédit.

L'approche par le territoire suppose alors de connaître les mécanismes d'entrée dans le territoire et de déploiement sur le territoire de l'offre (ici en microfinance) d'un opérateur. Ces choix peuvent relever de différentes appréciations. Mais ils témoignent d'un projet défini par et pour le territoire d'intervention.

Reprenons deux exemples qui témoignent de cette conception.

Opportunity Microcredit Romania est une société de microfinance établie en 1995 en Roumanie sous le statut d'Organisation Non Gouvernementale. Il s'agissait au départ d'une Fondation: Fundaţia Izvor, qui proposait un microcrédit pour les petits entrepreneurs dans le contexte d'un marché encore jeune et non préparé à satisfaire leur besoins. Les différents contextes législatifs ont conduit à un changement de nom et de structure: d'abord en Banque Populaire Izvor («Source» en roumain) (1995), puis en Société Commerciale Opportunity Microcredit România (2000) et enfin en Société de Microfinance Opportunity Microcredit România S.A. (2005).

Ses actionnaires sont le réseau Opportunity International, l'Association Izvor ou Ö̈kocredit et des organismes de crédit comme CoopEST, Citi, EBRD, Efse, responsAbility ou Symbiotics. Opportunity Microcredit Romania exerce son activité dans 8 départements de Transylvanie. Son siège est situé à Târgu Mures, ville universitaire abritant l'une des plus fortes communautés magyares du pays. Ce choix n'est pas anodin: Opportunity International s'était implanté en Hongrie préalablement à son implantation en Roumanie. La proximité linguistique et culturelle, des facilités à pouvoir déployer le modèle économique d'Opportunity, ont joué dans le choix d'implantation, tout comme la relative centralité (à l'échelle de la Roumanie entière) de la ville.

L'objectif d'Opportunity est de permettre de créer des emplois, d'améliorer les conditions de vie d'entrepreneurs issus de milieux désavantagés et d'assurer le soutien à l'égalité de chances au travers d'un partenariat avec les petits et tous petits entrepreneurs de Transylvanie, actifs dans les secteurs de production, des services, du commerce et d'agriculture. La sélection de ces microentrepreneurs relève de critères de bonne réputation au niveau local et d'un potentiel de croissance avéré, en l'absence d'accès aux services bancaires commerciaux. Bien entendu, d'autres conditions jouent comme la nature et le poids des ressources propres, les montants de profits dégagés par l'activité avant octroi du microcrédit, et le potentiel de création d'emploi.

Preuve de son implantation et de la relative reconnaissance de son activité, Opportunity est le premier établissement non-bancaire ayant reçu des fonds de la BRED pour le portage de microcrédits en Roumanie (2.8 Mds € en janvier 2007, selon le site wall-street.ro, 30.01.2007). Actuellement la société développe des projets de soutien à l'entrepreneuriat et de formation, anime des réseaux locaux d'entrepreneurs et entend 
favoriser le «consommer local»: ainsi, elle travaille à la création d'une communauté rassemblant de petits entrepreneurs et leurs clients dans le département de Bistrita, à dominante rurale, au nord de la Transylvanie.

Par son développement, cette société indique des choix d'implantation dans le territoire roumain et d'orientation de ses activités sur le territoire, donc de territorialisation, qui relèvent d'une politique globale axée sur une approche de social business. Il est bien un projet social, mais les choix de déploiement de l'activité sont induits à des degrés divers par les différentes formes de proximités.

L'autre exemple pouvant être avancé est celui de l'association française La Bonne Etoile (Briançon). Cette association loi 1901 créée en 2001, porte un microcrédit (BancSol) en partenariat avec l'association roumaine Montan Initiativ. Le projet d'association consiste à soutenir des initiatives dans les villages de piémont reculés des axes principaux, qui peu à peu se vident de leurs populations les plus jeunes.

Le microcrédit BancSol est jusqu'en 2011 proposé dans le seul département de Prahova, au sein duquel de nombreuses associations françaises ont développé des coopérations interassociatives et des communes des jumelages. Ces coopérations sont notamment nées à la fin des années 1980 de l'Opération Villages Roumains (OVR), qui consistait à établir des parainnages pour venir en aide aux villages menacés de destructions par la politique de systématisation développée par Nicolae Ceaucescu. La menace est passée mais le réseau s'est entretemps restructuré: d'abord à travers l'aide d'urgence du début des années 1990, et aujourd'hui au travers de projets de partenariats culturels, scolaires, etc. OVR anime un réseau d'agrotourisme à travers l'ensemble du pays et teste donc, via BancSol, un premier microcrédit dans le pays.

A partir de 2012, des initiatives de microcrédit vont être déployées dans d'autres départements de Roumanie, toujours au profit de régions rurales isolées, notamment dans les piémonts carpatiques ou des Monts Apuseni. Les villages potentiellement bénéficiaires sont sélectionnés au sein du réseau OVR et de ses associations partenaires: ce sont les préexistences de liens entre associations ou communes françaises, et leurs homologues roumaines, qui ici constituent le critère de choix d'implantation de l'offre. Les critères d'attribution de microcrédits aux personnes bénéficiaires relèvent ensuite des modalités de sélection d'usage pour tout microcrédit.

Mais l'on voit au travers de cet exemple que ce qui joue ici ne sont pas tellement des projets d'entreprises au départ mais bien des projets de société et des solidarités préinstituées. Notons que ce réseau partenarial s'inscrit là où ne vont pas - ou moins les organismes de microfinance identifiés par ailleurs en Roumanie: dans ces secteurs ruraux peu attractifs. Nous sommes face à un modèle d'économie solidaire.

Pour terminer, ce qui témoigne de dynamiques institutionnelles nous renvoie, aussi, à des caractéristiques propres aux lieux de diffusion du microcrédit, ou à l'inverse aux creux de l'offre (Glémain et al., 2010). L'offre peut être proposée en un lieu donné pour différentes raisons, que nous essayons ici d'expliquer par l'approche par les proximités (Pecqueur, Zimmerman, 2004):

- l'interconnaissance préalable comme dans le cas des réseaux nés de la proximité institutionnelle entre des communes roumaines et des mouvements associatifs (dans OVR notamment) ou des communes non roumaines (par jumelages), 
- un ancrage culturel ou idéologique qui se répercute dans des choix de promotion du microcrédit auprès de groupes ethnoculturels, linguistiques ou confessionnels spécifiques, voire auprès de certains corps de métiers ou certaines catégories de populations (en lien avec les niveaux de revenus) qui traduit dans une certaine mesure une proximité organisationnelle: il n'y a pas nécessairement d'interconnaissance préalable, mais un rapprochement s'effectue au travers d'un projet pour partie commun,

- enfin, pour des raisons qui tiennent de façon simplifiée au choix d'implantation d'un organisme en un lieu et dans un territoire donné - choix qui peut être induit par de multiples et diverses raisons - qui tend à favoriser les villes les plus importantes, attractives à la fois pour les salariés de l'organisation promouvant l'offre de microcrédit et pour les entreprises ou les personnes susceptibles de bénéficier de cette offre. Cela induit une proximité spatiale ou géographique.

\section{BIBLIOGRAPHIE}

BIOTEAU E., 2005, 'Des frontières et des hommes. Approche des recompositions territoriales frontalières', Thèse de Doctorat en Géographie, Université d'Angers, $418 \mathrm{p}$.

BOLTANSKI L. et THEVENOT L., 1991, De la justification. Les économies de la grandeur. Paris, Gallimard.

BOULINEAU E. et SUCIU M., 2008, Décentralisation et régionalisation en Bulgarie et en Roumanie. Les ambiguïtés de l'européanisation, dans L'espace géographique, Berlin, p. 349-363.

CAPELLE-POGACEAN A. et MICHEL P., PACE E. (dir.), 2008, Religion(s) et identité(s) en Europe. L'épreuve du pluriel. Paris, Presses de Sciences Po, 344 p.

CAPELLE-POGACEAN A. et RAGARU N., 2008, «En quoi les "partis ethniques" sontils "ethniques" ? Les trajectoires du MDL en Bulgarie et de l'UDMR en Roumanie», Questions de recherche, $\mathrm{n}^{\circ} 25$, juin 2008.

EUROM, 2010, Benchmarking Microfinance in Romania 2008-2009. Rapport pour le Réseau Européen de Microfinance, Londres.

DU GRANRUT C., 1996, Europe, le temps des Régions. Paris, LGDJ

GILBERT G., 1996, «Le fédéralisme financier. Perspectives de microéconomie spatiale», Revue Economique, 47, 2, 311-363.

GLEMAIN et al., 2010, Les territoires des finances solidaires. Une analyse en régions Bretagne et Pays-de-la-Loire. Paris, L'Harmattan, Coll. L'Esprit économique.

GLEMAIN P., 2000, 'Financement, croissance endogène, régionalisation et développement', Thèse de Doctorat en Sciences Economiques. Université de Nantes LEN-CORRAIL, sous la direction Pr. J-P. Gourlaouen.

IONESCU I., 2007, «La question de la pauvreté en Roumanie», Pensée plurielle, 2007/3, $16,141-151$.

MANOURY L., 2001, «L'opportunité d'un nouveau type de société à vocation sociale: le société coopérative d'interêt collectif», RECMA, 281, 108-134. 
MAUCOURANT J., 2011, Avez-vous lu Polanyi? Paris, Flammarion.

PECQUEUR B. et ZIMMERMAN J-B., 2004, Economie de proximités, Hermès-Lavoisier, $264 \mathrm{p}$.

PONSARD J-P., 2004, «Formalisation des connaissances, apprentissage organisationnel et rationalité interactive» p. 193-209, dans Orlean A (dir.), Analyse économique des conventions. Paris, PUF-Quadrige.

POLANYI K., 1983, La Grande Transformation. Paris, Gallimard.

REY V. et al., 2007, Atlas de la Roumanie. Montpellier, CNRS-GDRE S4/Paris, La Documentation française, 208 p., et [en ligne, langue roumaine : mdlpl.ro/documente/ atlas/a_asezari.htm]

RICOEUR-NICOLAÏ N., AUZOUY B., LAKHOUA F., MEUNIER N. et MIOTTI L., 1999, «Vers un élargissement sélectif de l’Union Européenne. Dix pays, deux cercles», Revue Economique, 50, 6, 1101-1122.

SAMOLYK K-A., 1994, «Banking conditions and regional economic perfeormance, evidence of a regional credit channel», Journal of Monetary Economics, 34-1, 259-278.

SECHET R. et KEERLE R., 2009, «Petite histoire des délicatesses de «l'équipe-degéographie-sociale-de-la-France-de-l'Ouest» avec le territoire», pp. 83-93, in Vanier M. (dir.), Territoires, territorialité, territorialisation. Controverses et perspectives, Presses Universitaires de Rennes, coll. Espace et Territoires.

SEUROT F., 1989, Le système économique de l'URSS. Paris, PUF.

TAUPIN M-T et GLEMAIN P., 2007, «Les logiques d'acteurs des finances solidaires contemporaine: de la résistance à la résilience?», Annals of Public and Cooperative Economics, 78, 4, 629-661.

TIRASPOLSKY A. et WILD G., 1992, Economies de l'Est en transition: critères de comparaison. Travaux de recherche de l'IFRI. Paris, Masson.

VANIER M. (dir.), 2009, Territoires, territorialité, territorialisation. Controverses et perspectives, Presses Universitaires de Rennes, coll. Espace et Territoires. 